\title{
Convergence Rates for a Reaction-Diffusion System
}

\author{
M. Kirane and N.-e. Tatar
}

\begin{abstract}
A class of reaction-diffusion systems is investigated. This class is motivated by some diffusive epidemic models, which serve to modelise the spread of Feline Immunodeficiency Virus (FIV) in the cat population, and sexually transmitted diseases. We obtain exponential convergence rates for a system with unbounded time dependent coefficients.
\end{abstract}

Keywords: Reaction-diffusion systems, asymptotic behavior, biomathematics

AMS subject classification: 35K57, 35B40, 92B05

\section{Introduction}

We consider the problem

$$
\begin{array}{lll}
u_{t}=d_{1} \Delta u-r_{1}(t) f_{1}(u) w^{\gamma}-r_{2}(t) f_{2}(u) z^{\eta} & & (x \in \Omega, t>0) \\
w_{t}=d_{2} \Delta w+r_{1}(t) f_{1}(u) w^{\gamma}+r_{2}(t) f_{2}(u) z^{\eta}-a w & & (x \in \Omega, t>0) \\
v_{t}=d_{3} \Delta v-r_{3}(t) f_{3}(v) w^{\sigma}-r_{4}(t) f_{4}(v) z^{\rho} & & (x \in \Omega, t>0) \\
z_{t}=d_{4} \Delta z+r_{3}(t) f_{3}(v) w^{\sigma}+r_{4}(t) f_{4}(v) z^{\rho}-a z & & (x \in \Omega, t>0) \\
\frac{\partial u}{\partial \nu}=\frac{\partial w}{\partial \nu}=\frac{\partial v}{\partial \nu}=\frac{\partial z}{\partial \nu}=0 & & (x \in \partial \Omega, t>0) \\
(u, w, v, z)(x, 0)=\left(u_{0}, w_{0}, v_{0}, z_{0}\right)(x) & & (x \in \Omega)
\end{array}
$$

where

- $\Omega$ is a bounded region in $\mathbb{R}^{N}$ with smooth boundary $\partial \Omega$

- the diffusion coefficients $d_{i}(i=1,2,3,4)$ and $a$ are positive constants

- the functions $r_{i}$ are of the form $r_{i}(t)=t^{k_{i}} g_{i}(t)(i=1,2,3,4)$

- $k_{i} \geq 0(i=1,2,3,4)$ and $g_{i}$ are continuous functions in $L^{2}(0, \infty)$

- the exponents $\gamma, \eta, \sigma, \rho$ are greater than one

- $f_{i}(i=1,2,3,4)$ are given functions to be precised later

- $\frac{\partial}{\partial \nu}$ denotes the outward normal derivative on $\partial \Omega$

- $\left(u_{0}, w_{0}, v_{0}, z_{0}\right)(x)$ is a given initial value of the solution.

M. Kirane: Univ. de Picardie, Fac. de Math. \& Inform., LAMFA FRE 2270, 33 rue Saint Leu, 80039 Amiens Cedex 1, France

N.-e. Tatar: Univ. Badji Mokhtar, Inst. de Math., B.P. 12, 23000 Annaba, Algérie 
We will study global existence, asymptotic behavior and convergence rates for solutions of problem (1).

If $f_{i}(y)=y(i=1,2,3,4)$ and $\gamma=\eta=\sigma=\rho=1$, then we find the problem studied by Fitzgibbon et al. [2]. This system modelises the circulation of a disease within a population which is confined to a bounded region. More precisely, it describes the spread of Feline Immunodeficiency Virus (FIV) in the cat population or sexually transmitted disease. The entities $u, w, v, z$ represent the susceptible males, infective males, susceptible females and infective females, respectively. See [2] and references therein for further explanations and related problems. In fact, in [2] the authors considered the system with age dependence and arrived at the conclusion that FIV (for the proposed model) is not endemic unless a significant source of new susceptibles is inserted in the system.

The following problem similar (in some sense) to ours was studied in [6] by $\mathrm{H}$. Hoshino:

$$
\begin{array}{ll}
S_{t}=d_{1} \Delta S-f(S) I^{m} & (x \in \Omega, t>0) \\
I_{t}=d_{2} \Delta I+f(S) I^{m}-r I^{n} & (x \in \Omega, t>0) \\
R_{t}=d_{3} \Delta R+r I^{n} & (x \in \Omega, t>0) \\
\frac{\partial S}{\partial \nu}=\frac{\partial I}{\partial \nu}=\frac{\partial R}{\partial \nu}=0 & (x \in \partial \Omega, t>0) \\
(S, I, R)(x, 0)=\left(S_{0}, I_{0}, R_{0}\right)(x) & (x \in \Omega)
\end{array}
$$

The author considered the case $1 \leq n \leq m$ and proved exponential convergence of the solution to $\left(S_{\infty}, 0, R_{\infty}\right)$ (the equilibrium state) when $n=1$ and polynomial convergence when $n>1$. The convergence is shown in $C^{\mu}(\bar{\Omega})^{3}$ with $\mu \in[0,2)$.

In our case, we use ideas from [9 - 11] to study convergence rates for solutions of problem (1). We should mention that global existence and convergence of solutions present no new difficulties with respect to previous results. However, the arguments in [6] leading to exponential decay are no longer valid.

\section{Notation and preliminaries}

Let $W^{l, p}(\Omega)$ denotes the usual Sobolev space of order $l \geq 0$ for $1 \leq p \leq \infty$; \|\|$_{p}$ denotes the norm of $L^{p}(\Omega)$. The space $C^{\sigma}(\bar{\Omega})(\sigma \geq 0)$ is the Banach space of $[\sigma]$ times continuously differentiable functions in $\bar{\Omega}$ whose $[\sigma]$-th order derivatives are Hölder continuous with exponent $\sigma-[\sigma]$, so that $C^{0}(\bar{\Omega})=C(\bar{\Omega})$ and $C^{1}(\bar{\Omega})$ are the Banach spaces of continuous and of continuously differentiable functions in $\bar{\Omega}$, respectively.

Definition 2.1. For $p \in(1, \infty)$ we define

$$
\begin{aligned}
D\left(A_{p}\right)=D\left(B_{p}\right)=D\left(F_{p}\right)=D\left(G_{p}\right) & =\left\{y \in W^{2, p}(\Omega):\left.\frac{\partial y}{\partial \nu}\right|_{\partial \Omega}=0\right\} \\
A_{p} y & =-d_{1} \Delta y \\
B_{p} y & =-\left(d_{2} \Delta-a\right) y \\
F_{p} y & =-d_{3} \Delta y \\
G_{p} y & =-\left(d_{4} \Delta-a\right) y .
\end{aligned}
$$


Then, for $p \in(1, \infty),-A_{p},-B_{p},-F_{p},-G_{p}$ are sectorial operators (see [5]) and generating analytic semigroups $\left\{e^{-t A_{p}}\right\}_{t \geq 0},\left\{e^{-t B_{p}}\right\}_{t \geq 0},\left\{e^{-t F_{p}}\right\}_{t \geq 0},\left\{e^{-t G_{p}}\right\}_{t \geq 0}$ in $L^{p}(\Omega)$, respectively.

Definition 2.2. We shall write for $y \in L^{k}(\Omega)$ with $k \in(1, \infty)$

$$
Q_{0} y=|\Omega|^{-1} \int_{\Omega} y(x) d x \quad \text { and } \quad Q_{+} y(x)=y(x)-Q_{0} y
$$

where $|\Omega|$ is the volume of $\Omega$.

Definition 2.3. $A_{p+}=\left.A_{p}\right|_{Q_{+} L^{p}(\Omega)}$ and $F_{p+}=\left.F_{p}\right|_{Q_{+} L^{p}(\Omega)}$ will denote the restriction of $A_{p}$ and $F_{p}$, respectively, onto $Q_{+} L^{p}(\Omega)$.

It is then clear that for $p \in(1, \infty)$ the operators $A_{p+}$ and $F_{p+}$ generate analytic semigroups $\left\{e^{-t A_{p+}}\right\}_{t \geq 0}$ and $\left\{e^{-t F_{p+}}\right\}_{t \geq 0}$, respectively. The fractional powers of the above operators are defined in the usual way (see [5]). In fact, we will need the fractional powers of the operators $A_{p+}, B_{p}, F_{p+}$ and $G_{p}$.

Next, we prepare some lemmas that will be needed in the sequel.

Lemma 2.1. Let $A$ be a sectorial operator in $X=L^{p}(\Omega)(1 \leq p<\infty)$ with $D(A)=X^{1} \hookrightarrow W^{m, p}(\Omega)$ for some $m \geq 1$. Then, for $0 \leq \alpha \leq 1, X^{\alpha} \hookrightarrow C^{\nu}$ when $0 \leq \nu<m \alpha-\frac{N}{p}$.

Lemma 2.2. Let $\lambda$ denote the least positive eigenvalue of the Laplacian with homogeneous Neumann boundary condition and $p \in(1, \infty)$. For every $\alpha \in[0,1)$, there exist constants $C_{i}>0(i=1,2,3,4)$ such that for $t>0$ and $y \in L^{p}(\Omega)$

$$
\begin{aligned}
\left\|A_{p+}^{\alpha} e^{-t A_{p+}} Q_{+} y\right\|_{p} & \leq C_{1} t^{-\alpha} e^{-d_{1} \lambda t}\left\|Q_{+} y\right\|_{p} \\
\left\|B_{p}^{\alpha} e^{-t B_{p}} y\right\|_{p} & \leq C_{2} t^{-\alpha} e^{-a t}\|y\|_{p} \\
\left\|F_{p+}^{\alpha} e^{-t F_{p+}} y\right\|_{p} & \leq C_{3} t^{-\alpha} e^{-d_{3} \lambda t}\left\|Q_{+} y\right\|_{p} \\
\left\|G_{p}^{\alpha} e^{-t G_{p}} y\right\|_{p} & \leq C_{4} t^{-\alpha} e^{-a t}\|y\|_{p} .
\end{aligned}
$$

Lemma 2.3. Let $p \in(1, \infty), m \geq 1$ and $\alpha \in[0,1)$. Then there exists a constant $C>0$ such that

$$
\|y\|_{p m} \leq C\left\|B_{p}^{\alpha} y\right\|_{p}^{\theta}\|y\|_{p}^{1-\theta}
$$

where $\theta$ satisfies $\frac{N(m-1)}{2 p m \alpha}<\theta<1$.

See [5: Theorem 1.6.1], [5: p. 37/Gagliardo-Nirenberg inequality] and [5: Theorem 1.4.4], respectively, for the proofs of Lemmas $2.1-2.3$.

Lemma 2.4. Let $\alpha \in[0,1)$ and $\beta \in \mathbb{R}$. There exists a constant $C=C(\alpha, \beta)>0$ such that

$$
\int_{0}^{t} s^{-\alpha} e^{\beta s} d s \leq \begin{cases}C e^{\beta t} & \text { if } \beta>0 \\ C(t+1) & \text { if } \beta=0 \\ C & \text { if } \beta<0\end{cases}
$$

See [7] for the proof of this lemma. 
Lemma 2.5. If $\mu, \nu, \tau, z>0$, then

$$
z^{1-\nu} \int_{0}^{z}(z-\xi)^{\nu-1} \xi^{\mu-1} e^{-\tau \xi} d \xi \leq C \tau^{-\mu}
$$

where $C$ is a constant independent of $z$.

See [9] or [12: p. 23/Proposition 2.1] for the proof of this lemma.

Lemma 2.6. Let $a, b, K, \psi$ be non-negative continuous functions on the interval $I=(0, T) \quad(0<T \leq \infty)$, let $\omega:(0, \infty) \rightarrow \mathbb{R}$ be a continuous, non-negative and nondecreasing function with $\omega(0)=0$ and $\omega(u)>0$ for $u>0$, and let $A(t)=\max _{0 \leq s \leq t} a(s)$ and $B(t)=\max _{0 \leq s \leq t} b(s)$. Assume that

$$
\psi(t) \leq a(t)+b(t) \int_{0}^{t} K(s) \omega(\psi(s)) d s \quad(t \in I) .
$$

Then

$$
\psi(t) \leq H^{-1}\left[H(A(t))+B(t) \int_{0}^{t} K(s) d s\right] \quad\left(t \in\left(0, T_{1}\right)\right)
$$

where $H(v)=\int_{v_{0}}^{v} \frac{d \tau}{\omega(\tau)}\left(v \geq v_{0}>0\right), H^{-1}$ is the inverse of $H$ and $T_{1}>0$ is such that $H(A(t))+B(t) \int_{0}^{t} K(s) d s \in D\left(H^{-1}\right)$ for all $t \in\left(0, T_{1}\right)$.

See [1] for the proof of this lemma.

\section{Existence and asymptotic behavior}

In this section we shall state without proofs some results on the existence and asymptotic behavior of solutions of problem (1). Let us first make the following assumptions:

(i) $\left\|u_{0}\right\|_{1},\left\|w_{0}\right\|_{1},\left\|v_{0}\right\|_{1},\left\|z_{0}\right\|_{1}>0$

(ii) $f_{i}(i=1,2,3,4)$ are non- negative $C^{1}$-functions on $[0, \infty)$

(iii) $f_{i}(0)=0$, and $f_{i}(y)>0$ if and only if $y>0(i=1,2,3,4)$

(iv) $1<\eta \leq \rho$ and $1<\sigma \leq \gamma$.

Theorem 3.1. Assume that $0 \leq u_{0}, w_{0}, v_{0}, z_{0} \in C(\bar{\Omega})$ and assumption $(\mathrm{H})$ hold. Then there exists a unique non-negative global solution $(u, w, v, z)$ to problem (1). Moreover, there exists a number $M>0$ such that

$$
0 \leq w(x, t), z(x, t) \leq M \quad(x \in \Omega, t \geq 0)
$$

and

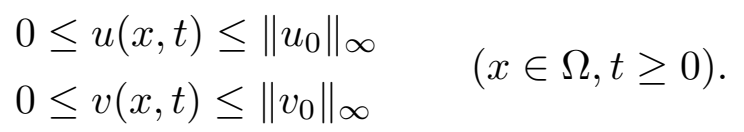

The local existence and positivity may be proved in a standard manner (see [5: Chapter 3]. The continuation of the solution follows from the uniform a priori boundedness. The boundedness of $u$ and $v$ follow from the maximum principle. As mentioned 
in [6] we may use the argument by Haraux and Youkana [4] to show that the positive functionals

$$
\int_{\Omega}\left[1+\delta_{1}\left(u+u^{2}\right)\right] e^{\varepsilon_{1} w} d x \quad \text { and } \quad \int_{\Omega}\left[1+\delta_{2}\left(v+v^{2}\right)\right] e^{\varepsilon_{2} z} d x
$$

are non-increasing on the maximal time interval for some constants $\delta_{i}(i=1,2)$ and $\varepsilon_{i}(i=1,2)$. This implies the boundedness of $w$ and $z$, that is (2). This argument works for a larger class of non-linearities. In fact, we may replace $w^{\gamma}, z^{\eta}, w^{\sigma}, z^{\rho}$ by $\varphi(w), \psi(z), \chi(w), \phi(z)$ accordingly, provided that

$$
\lim _{y \rightarrow+\infty} \frac{1}{y} \log (1+\varphi(y))=\lim _{y \rightarrow+\infty} \frac{1}{y} \log (1+\phi(y))=0
$$

and

$$
\|\psi(y)\|_{N} \leq\|\phi(y)\|_{N}, \quad\|\chi(y)\|_{N} \leq\|\varphi(y)\|_{N} .
$$

Theorem 3.2. Assume the hypotheses of Theorem 3.1 hold. Then, for every $\mu \in$ $[0,2)$,

$$
(u(t), w(t), v(t), z(t)) \rightarrow\left(u_{\infty}, 0, v_{\infty}, 0\right)
$$

in $C^{\mu}(\bar{\Omega})$ as $t \rightarrow+\infty$.

The proof is similar to that of [6: Theorem 2.2] (combined with the new Lemma 2.5) and is therefore omitted.

\section{Convergence rates}

In this section we state and prove our main theorem. Let us set

$$
\begin{aligned}
& m_{1}=\max \{\gamma-1, \eta-1, \sigma-1, \rho-1\} \\
& m_{2}=\max \{\gamma, \eta, \sigma, \rho\} .
\end{aligned}
$$

Observe that $m_{1}=m_{2}-1$ and from assumption $(\mathrm{H}) /(\mathrm{iv})$ we have $m_{2}=\max \{\gamma, \rho\}$. Let $q$ and $q^{*}$ be conjugate exponents, i.e. $\frac{1}{q}+\frac{1}{q^{*}}=1$, such that

$$
q^{*}= \begin{cases}\frac{2 y+1}{y} & \text { if } y \leq 1 \\ 2 & \text { if } y>1\end{cases}
$$

with $y=\frac{1-\alpha}{\alpha}$. We also set $h(t)=\max \left\{g_{i}^{q^{*}}(t): i=1,2,3,4\right\}$.

Theorem 4.1. Let the hypothesis of Theorem 3.1 hold. Suppose that $p>N \frac{m_{1}}{2}, \alpha>$ $N \frac{m_{1}}{2 p}, k_{i}>m_{2}-\frac{1}{q^{*}}(i=1,2,3,4)$ and

$$
\int_{0}^{+\infty} h(\tau) d \tau<M_{1}\left(\left\|w_{0}\right\|_{p}^{q^{*}}+\left\|z_{0}\right\|_{p}^{q^{*}}\right)^{-r}
$$


for some constants $M_{1}>0$ and $r>0$ (to be determined later). Then for every $\mu<$ $2 \alpha-\frac{N}{p}$ we have

$$
\|w(t)\|_{C^{\mu}(\bar{\Omega})},\|z(t)\|_{C^{\mu}(\bar{\Omega})} \leq M_{2} e^{-a t} \quad(t \rightarrow+\infty) .
$$

Moreover:

(a) If $\gamma, \eta>d_{1} \frac{\lambda}{a}$ and $\sigma, \rho>d_{3} \frac{\lambda}{a}$, then

$$
\left.\begin{array}{rl}
\left\|u(t)-u_{\infty}\right\|_{C^{\mu}(\bar{\Omega})} & \leq M_{3} e^{-\min \left\{a, d_{1} \lambda\right\} t} \\
\left\|v(t)-v_{\infty}\right\|_{C^{\mu}(\bar{\Omega})} & \leq M_{4} e^{-\min \left\{a, d_{3} \lambda\right\} t}
\end{array}\right\} \quad(t \rightarrow+\infty) .
$$

(b) If at least one of the assumptions in statement (a) fails, say $\gamma \leq d_{1} \frac{\lambda}{a}$, then assuming that

$$
\int_{0}^{t} e^{q^{*} b \tau} g_{1}^{q^{*}}(\tau) d \tau=O\left(e^{q^{*} \tilde{b} t}\right) \quad(t \rightarrow+\infty)
$$

for some $b>d_{1} \lambda-a \gamma$ and $\tilde{b}<d_{1} \lambda$, we get

$$
\left\|u(t)-u_{\infty}\right\|_{C^{\mu}(\bar{\Omega})} \leq M_{5} e^{-\min \left\{a, d_{1} \lambda-\tilde{b}\right\} t} \quad(t \rightarrow+\infty) .
$$

The other cases are treated similarly.

Proof. Let us consider the integral equations associated to $(1)_{2}$ and $(1)_{4}$

$$
\begin{aligned}
& w(t)=e^{-t B_{p}} w_{0}+\int_{0}^{t} e^{-(t-\tau) B_{p}}\left\{r_{1}(\tau) f_{1}(u) w^{\gamma}+r_{2}(\tau) f_{2}(u) z^{\eta}\right\} d \tau \\
& z(t)=e^{-t G_{p}} z_{0}+\int_{0}^{t} e^{-(t-\tau) G_{p}}\left\{r_{3}(\tau) f_{3}(v) w^{\sigma}+r_{4}(\tau) f_{4}(v) z^{\rho}\right\} d \tau .
\end{aligned}
$$

Using Lemma 2.2 we can see that

$$
\begin{aligned}
\left\|B_{p}^{\alpha} w\right\|_{p} \leq & C_{2}\left\{t^{-\alpha} e^{-a t}\left\|w_{0}\right\|_{p}\right. \\
& \left.+\int_{0}^{t}(t-\tau)^{-\alpha} e^{-a(t-\tau)}\left[\tau^{k_{1}} g_{1}(\tau) f_{1}(u) w^{\gamma}+\tau^{k_{2}} g_{2}(\tau) f_{2}(u) z^{\eta}\right] d \tau\right\} \\
\left\|G_{p}^{\alpha} z\right\|_{p} \leq & C_{4}\left\{t^{-\alpha} e^{-a t}\left\|z_{0}\right\|_{p}\right. \\
& \left.+\int_{0}^{t}(t-\tau)^{-\alpha} e^{-a(t-\tau)}\left[\tau^{k_{3}} g_{3}(\tau) f_{3}(v) w^{\sigma}+\tau^{k_{4}} g_{4}(\tau) f_{4}(v) z^{\rho}\right] d \tau\right\} .
\end{aligned}
$$

Multiplying both sides of (4) - (5) by $e^{a t}$ and taking into account assumption (H)/(ii) and (3) we find

$$
\begin{aligned}
e^{a t}\left\|B_{p}^{\alpha} w\right\|_{p} \leq & C_{2}\left\{t^{-\alpha}\left\|w_{0}\right\|_{p}+\left\|f_{1}(u)\right\|_{\infty} \int_{0}^{t}(t-\tau)^{-\alpha} e^{a \tau} \tau^{k_{1}} g_{1}(\tau)\left\|w^{\gamma}\right\|_{p} d \tau\right. \\
& \left.+\left\|f_{2}(u)\right\|_{\infty} \int_{0}^{t}(t-\tau)^{-\alpha} e^{a \tau} \tau^{k_{2}} g_{2}(\tau)\left\|z^{\eta}\right\|_{p} d \tau\right\} \\
e^{a t}\left\|G_{p}^{\alpha} z\right\|_{p} \leq & C_{4}\left\{t^{-\alpha}\left\|z_{0}\right\|_{p}+\left\|f_{3}(v)\right\|_{\infty} \int_{0}^{t}(t-\tau)^{-\alpha} e^{a \tau} \tau^{k_{3}} g_{3}(\tau)\left\|w^{\sigma}\right\|_{p} d \tau\right. \\
& \left.+\left\|f_{4}(v)\right\|_{\infty} \int_{0}^{t}(t-\tau)^{-\alpha} e^{a \tau} \tau^{k_{4}} g_{4}(\tau)\left\|z^{\rho}\right\|_{p} d \tau\right\}
\end{aligned}
$$


Let $1<l<\min \{\gamma, \eta, \sigma, \rho\}=\min \{\eta, \sigma\}$. We have by Lemma 2.3

$$
\left\|w^{\gamma}\right\|_{p}=\|w\|_{\gamma p}^{\gamma} \leq C_{5}\left\|B_{p}^{\alpha} w\right\|_{p}^{\theta_{1} \gamma}\|w\|_{p}^{\left(1-\theta_{1}\right) \gamma} .
$$

Choose $\theta_{1}$ such that $\theta_{1} \gamma=l$, i.e. $\theta_{1}=\frac{l}{\gamma}$. This choice is possible by our assumptions on $p$ and $\alpha$. Then

$$
\left\|w^{\gamma}\right\|_{p} \leq C_{6}\left\|B_{p}^{\alpha} w\right\|_{p}^{l}
$$

Also,

$$
\left\|z^{\eta}\right\|_{p}=\|z\|_{\eta p}^{\eta} \leq C_{7}\left\|G_{p}^{\alpha} z\right\|_{p}^{\theta_{2} \eta}\|z\|_{p}^{\left(1-\theta_{2}\right) \eta} .
$$

Choosing $\theta_{2}$ such that $\theta_{1} \eta=l$, i.e. $\theta_{2}=\frac{l}{\eta}$, we find

$$
\left\|z^{\eta}\right\|_{p} \leq C_{8}\left\|G_{p}^{\alpha} z\right\|_{p}^{l}
$$

In the same fashion we obtain $\left\|w^{\sigma}\right\|_{p} \leq C_{9}\left\|B_{p}^{\alpha} w\right\|_{p}^{l}$ and $\left\|z^{\rho}\right\|_{p} \leq C_{10}\left\|G_{p}^{\alpha} z\right\|_{p}^{l}$. Using these relations in (6) - (7) it appears that

$$
\begin{aligned}
e^{a t}\left\|B_{p}^{\alpha} w\right\|_{p} \leq & C_{11}\left\{t^{-\alpha}\left\|w_{0}\right\|_{p}+\int_{0}^{t}(t-\tau)^{-\alpha} e^{a \tau} e^{-l a \tau} e^{l a \tau} \tau^{k_{1}} g_{1}(\tau)\left\|B_{p}^{\alpha} w\right\|_{p}^{l} d \tau\right. \\
& \left.+\int_{0}^{t}(t-\tau)^{-\alpha} e^{a \tau} e^{-l a \tau} e^{l a \tau} \tau^{k_{2}} g_{2}(\tau)\left\|G_{p}^{\alpha} z\right\|_{p}^{l} d \tau\right\} \\
e^{a t}\left\|G_{p}^{\alpha} z\right\|_{p} \leq & C_{12}\left\{t^{-\alpha}\left\|z_{0}\right\|_{p}+\int_{0}^{t}(t-\tau)^{-\alpha} e^{a \tau} e^{-l a \tau} e^{l a \tau} \tau^{k_{3}} g_{3}(\tau)\left\|B_{p}^{\alpha} w\right\|_{p}^{l} d \tau\right. \\
& \left.+\int_{0}^{t}(t-\tau)^{-\alpha} e^{a \tau} e^{-l a \tau} e^{l a \tau} \tau^{k_{4}} g_{4}(\tau)\left\|G_{p}^{\alpha} z\right\|_{p}^{l} d \tau\right\} .
\end{aligned}
$$

Let us now set

$$
\begin{aligned}
E_{w}(t) & =e^{a t} t^{\alpha}\left\|B_{p}^{\alpha} w\right\|_{p} \\
E_{z}(t) & =e^{a t} t^{\alpha}\left\|G_{p}^{\alpha} z\right\|_{p} .
\end{aligned}
$$

We see that

$$
\begin{aligned}
E_{w}(t) \leq & C_{11}\left\{\left\|w_{0}\right\|_{p}+t^{\alpha} \int_{0}^{t}(t-\tau)^{-\alpha} e^{a(1-l) \tau} \tau^{k_{1}-\alpha l} g_{1}(\tau) E_{w}^{l}(\tau) d \tau\right. \\
& \left.+t^{\alpha} \int_{0}^{t}(t-\tau)^{-\alpha} e^{a(1-l) \tau} \tau^{k_{2}-\alpha l} g_{2}(\tau) E_{z}^{l}(\tau) d \tau\right\} \\
E_{z}(t) \leq & C_{12}\left\{\left\|z_{0}\right\|_{p}+t^{\alpha} \int_{0}^{t}(t-\tau)^{-\alpha} e^{a(1-l) \tau} \tau^{k_{3}-\alpha l} g_{3}(\tau) E_{w}^{l}(\tau) d \tau\right. \\
& \left.+t^{\alpha} \int_{0}^{t}(t-\tau)^{-\alpha} e^{a(1-l) \tau} \tau^{k_{4}-\alpha l} g_{4}(\tau) E_{z}^{l}(\tau) d \tau\right\} .
\end{aligned}
$$

Let us estimate the integrals $I_{1}, I_{2}$ in (8) and $I_{3}, I_{4}$ in (9) separately. 
(i) Suppose that $y>1$. Then $1-2 \alpha>0$, i.e. $\alpha<\frac{1}{2}$. Applying the Cauchy-Schwarz inequality we get

$$
I_{1} \leq\left(\int_{0}^{t}(t-\tau)^{-2 \alpha} e^{2 a(1-l) \tau} \tau^{2\left(k_{1}-\alpha l\right)} d \tau\right)^{\frac{1}{2}}\left(\int_{0}^{t} g_{1}^{2}(\tau) E_{w}^{2 l}(\tau) d \tau\right)^{\frac{1}{2}} .
$$

As $1+2\left(k_{1}-\alpha l\right)>0$ holds from the assumption $k_{1}>m_{2}-\frac{1}{2}$, Lemma 2.5 implies

$$
I_{1} \leq K_{1} t^{-\alpha}\left(\int_{0}^{t} g_{1}^{2}(\tau) E_{w}^{2 l}(\tau) d \tau\right)^{\frac{1}{2}}
$$

In the same manner we obtain

$$
\begin{aligned}
& I_{2} \leq K_{2} t^{-\alpha}\left(\int_{0}^{t} g_{2}^{2}(\tau) E_{z}^{2 l}(\tau) d \tau\right)^{\frac{1}{2}} \\
& I_{3} \leq K_{3} t^{-\alpha}\left(\int_{0}^{t} g_{3}^{2}(\tau) E_{w}^{2 l}(\tau) d \tau\right)^{\frac{1}{2}} \\
& I_{4} \leq K_{4} t^{-\alpha}\left(\int_{0}^{t} g_{4}^{2}(\tau) E_{z}^{2 l}(\tau) d \tau\right)^{\frac{1}{2}} .
\end{aligned}
$$

Substituting estimates (10) - (13) into (8) - (9) we find

$$
\begin{aligned}
& E_{w}(t) \leq C_{13}\left\{\left\|w_{0}\right\|_{p}+\left(\int_{0}^{t} g_{1}^{2}(\tau) E_{w}^{2 l}(\tau) d \tau\right)^{\frac{1}{2}}+\left(\int_{0}^{t} g_{2}^{2}(\tau) E_{z}^{2 l}(\tau) d \tau\right)^{\frac{1}{2}}\right\} \\
& E_{z}(t) \leq C_{14}\left\{\left\|z_{0}\right\|_{p}+\left(\int_{0}^{t} g_{3}^{2}(\tau) E_{w}^{2 l}(\tau) d \tau\right)^{\frac{1}{2}}+\left(\int_{0}^{t} g_{4}^{2}(\tau) E_{z}^{2 l}(\tau) d \tau\right)^{\frac{1}{2}}\right\}
\end{aligned}
$$

Therefore

$$
\begin{aligned}
& E_{w}^{2}(t) \leq C_{15}\left\{\left\|w_{0}\right\|_{p}^{2}+\int_{0}^{t} g_{1}^{2}(\tau) E_{w}^{2 l}(\tau) d \tau+\int_{0}^{t} g_{2}^{2}(\tau) E_{z}^{2 l}(\tau) d \tau\right\} \\
& E_{z}^{2}(t) \leq C_{16}\left\{\left\|z_{0}\right\|_{p}^{2}+\int_{0}^{t} g_{3}^{2}(\tau) E_{w}^{2 l}(\tau) d \tau+\int_{0}^{t} g_{4}^{2}(\tau) E_{z}^{2 l}(\tau) d \tau\right\}
\end{aligned}
$$

Summing up (14) and (15) with $F_{w}=E_{w}^{2}$ and $F_{z}=E_{z}^{2}$, it results that

$$
\begin{gathered}
F(t) \equiv F_{w}(t)+F_{z}(t) \leq \\
C_{17}\left\{\left\|w_{0}\right\|_{p}^{2}+\left\|z_{0}\right\|_{p}^{2}+\int_{0}^{t}\left(g_{1}^{2}+g_{3}^{2}\right)(\tau) F_{w}^{l}(\tau) d \tau+\int_{0}^{t}\left(g_{2}^{2}+g_{4}^{2}\right)(\tau) F_{z}^{l}(\tau) d \tau\right\} .
\end{gathered}
$$

Hence

$$
F(t) \leq C_{18}\left\{\left\|w_{0}\right\|_{p}^{2}+\left\|z_{0}\right\|_{p}^{2}+\int_{0}^{t} h(\tau) F^{l}(\tau) d \tau\right\}
$$


where $h(t)=\max \left\{g_{i}^{2}(t): i=1,2,3,4\right\}$. From Lemma 2.6 we infer

$$
F(t) \leq H^{-1}\left[H\left\{C_{18}\left(\left\|w_{0}\right\|_{p}^{2}+\left\|z_{0}\right\|_{p}^{2}\right)\right\}+C_{18} \int_{0}^{t} h(\tau) d \tau\right]
$$

where $H(v)=\frac{v^{1-l}}{1-l}-\frac{v_{0}^{1-l}}{1-l}$ and $H^{-1}(z)=\left\{v_{0}^{1-l}-(l-1) z\right\}^{-\frac{1}{l-1}}$. Therefore

$$
F(t) \leq\left\{\left[C_{18}\left(\left\|w_{0}\right\|_{p}^{2}+\left\|z_{0}\right\|_{p}^{2}\right)\right]^{1-l}-(l-1) C_{18} \int_{0}^{t} h(\tau) d \tau\right\}^{-\frac{1}{l-1}} .
$$

The first assertion of the theorem follows.

Next, as in [6], we write $u(t)-u_{\infty}=\left(Q_{0} u(t)-u_{\infty}\right)+Q_{+} u(t)$ and estimate both terms on the right-hand side separately. Integrating the first equation $(1)_{1}$ over $[0, t] \times \Omega$, we see that by Green's formula

$$
\int_{\Omega} u(x, t) d x+\int_{0}^{t} \int_{\Omega}\left\{r_{1}(\tau) f_{1}(u) w^{\gamma}+r_{2}(\tau) f_{2}(u) z^{\eta}\right\} d x d \tau=\int_{\Omega} u_{0}(x) d x .
$$

Therefore,

$$
\left|Q_{0} u(t)-u_{\infty}\right|=|\Omega|^{-1}\left\{\int_{t}^{+\infty} \int_{\Omega}\left(r_{1}(\tau) f_{1}(u) w^{\gamma}+r_{2}(\tau) f_{2}(u) z^{\eta}\right) d x d \tau\right\} .
$$

From (3), assumption (H)/(ii) and the previous estimates of $w$ and $z$ it follows that

$$
\left|Q_{0} u(t)-u_{\infty}\right| \leq C\left\{\int_{t}^{+\infty} e^{-a \tau}\left(\tau^{k_{1}-\gamma \alpha} e^{-(\gamma-1) a \tau}+\tau^{k_{2}-\eta \alpha} e^{-(\eta-1) a \tau}\right) d \tau\right\}
$$

Here and below $C$ will denote a generic constant which may change from line to line. By the definition of the Euler gamma function it is clear that

$$
\left|Q_{0} u(t)-u_{\infty}\right| \leq C e^{-a t}\left[\Gamma^{\frac{1}{2}}\left(1+2\left(k_{1}-\gamma \alpha\right)\right)+\Gamma^{\frac{1}{2}}\left(1+2\left(k_{2}-\eta \alpha\right)\right)\right]
$$

for any $t \geq t_{1}>0$, provided that $1+2\left(k_{1}-\gamma \alpha\right)>0$ and $1+2\left(k_{2}-\eta \alpha\right)>0$. Note that these conditions are satisfied by our assumptions.

Concerning $Q_{+} u$, since we are working away from 0 , it is convenient to apply $A_{p+}^{\alpha} Q_{+}$ to the integral equation

$$
u(t)=e^{-\left(t-t_{1}\right) A_{p+}} u\left(t_{1}\right)-\int_{t_{1}}^{t} e^{-(t-\tau) A_{p+}}\left\{r_{1}(\tau) f_{1}(u) w^{\gamma}+r_{2}(\tau) f_{2}(u) z^{\eta}\right\} d \tau .
$$

Thus, for $t \geq t_{1}>0$ we have from Lemma 2.2

$$
\begin{aligned}
& \left\|A_{p+}^{\alpha} Q_{+} u(t)\right\|_{p} \\
& \leq C\left[e^{-d_{1} \lambda\left(t-t_{1}\right)}\left\|Q_{+} u\left(t_{1}\right)\right\|_{p}\right. \\
& \left.\quad+\int_{t_{1}}^{t}(t-\tau)^{-\alpha} e^{-d_{1} \lambda(t-\tau)}\left\|Q_{+}\left\{r_{1}(\tau) f_{1}(u) w^{\gamma}+r_{2}(\tau) f_{2}(u) z^{\eta}\right\}\right\|_{p} d \tau\right] .
\end{aligned}
$$


The boundedness of $u$ and assumption (H)/(ii) imply that

$$
\begin{aligned}
& \left\|A_{p+}^{\alpha} Q_{+} u(t)\right\|_{p} \\
& \leq C\left[e^{-d_{1} \lambda\left(t-t_{1}\right)}\left\|Q_{+} u\left(t_{1}\right)\right\|_{p}\right. \\
& \left.\quad+\int_{t_{1}}^{t}(t-\tau)^{-\alpha} e^{-d_{1} \lambda(t-\tau)}\left\|\left\{r_{1}(\tau)\|w\|_{\gamma p}^{\gamma}+r_{2}(\tau)\|z\|_{\eta p}^{\eta}\right\}\right\|_{p} d \tau\right] .
\end{aligned}
$$

Whence

$$
\begin{aligned}
& \left\|A_{p+}^{\alpha} Q_{+} u(t)\right\|_{p} \\
& \leq \quad C\left[e^{-d_{1} \lambda\left(t-t_{1}\right)}\left\|Q_{+} u\left(t_{1}\right)\right\|_{p}\right. \\
& \left.\quad+\int_{0}^{t-t_{1}}\left(t-t_{1}-\tau\right)^{-\alpha} e^{-d_{1} \lambda\left(t-t_{1}-\tau\right)}\left\{\tau^{k_{1}-\gamma \alpha} e^{-a \gamma \tau}+\tau^{k_{2}-\eta \alpha} e^{-a \eta \tau}\right\} d \tau\right] .
\end{aligned}
$$

As $k_{1}-\gamma \alpha>-1$ and $k_{2}-\eta \alpha>-1$ (by our assumptions) we may use Lemma 2.5 to get the following:

( $\alpha$ ) When $d_{1} \lambda-a \gamma<0$,

$$
\left\|A_{p+}^{\alpha} Q_{+} u(t)\right\|_{p} \leq C e^{-d_{1} \lambda\left(t-t_{1}\right)}\left\{\left\|Q_{+} u\left(t_{1}\right)\right\|_{p}+\left(t-t_{1}\right)^{-\alpha}\right\}
$$

and the first assertion in statement (a) follows. The second assertion may be proved similarly.

( $\beta)$ If $d_{1} \lambda-a \gamma \geq 0$, then the first integral in

$$
\begin{aligned}
& \left\|A_{p+}^{\alpha} Q_{+} u(t)\right\|_{p} \\
& \leq \quad C\left[e^{-d_{1} \lambda\left(t-t_{1}\right)}\left\|Q_{+} u\left(t_{1}\right)\right\|_{p}+e^{-d_{1} \lambda\left(t-t_{1}\right)} \int_{0}^{t-t_{1}}\left(t-t_{1}-\tau\right)^{-\alpha}\right. \\
& \left.\quad \times\left\{\tau^{k_{1}-\gamma \alpha} e^{\left(d_{1} \lambda-a \gamma\right) \tau} g_{1}(\tau)+\tau^{k_{2}-\eta \alpha} e^{\left(d_{1} \lambda-a \eta\right) \tau} g_{2}(\tau)\right\} d \tau\right]
\end{aligned}
$$

can be treated as

$$
\begin{aligned}
& \int_{0}^{t-t_{1}}\left(t-t_{1}-\tau\right)^{-\alpha} \tau^{k_{1}-\gamma \alpha} e^{\left(d_{1} \lambda-a \gamma\right) \tau} g_{1}(\tau) d \tau \\
& \quad \leq\left(\int_{0}^{t-t_{1}}\left(t-t_{1}-\tau\right)^{-2 \alpha} \tau^{2\left(k_{1}-\gamma \alpha\right)} e^{2\left[\left(d_{1} \lambda-a \gamma\right)-b\right] \tau} d \tau\right)^{\frac{1}{2}}\left(\int_{0}^{t-t_{1}} e^{2 b \tau} g_{1}^{2}(\tau) d \tau\right)^{\frac{1}{2}} \\
& \quad \leq C\left(t-t_{1}\right)^{-\alpha} e^{\tilde{b}\left(t-t_{1}\right)} .
\end{aligned}
$$

(ii) In the case $y \leq 1$, the argument is similar to that of the case (i) except we use Hölder's inequality instead of the Cauchy-Schwarz inequality. So, for instance,

$$
I_{1} \leq\left(\int_{0}^{t}(t-\tau)^{-q \alpha} e^{q a(1-l) \tau} \tau^{q\left(k_{1}-\alpha l\right)} d \tau\right)^{\frac{1}{q}}\left(\int_{0}^{t} g_{1}^{q^{*}}(\tau) E_{w}^{q^{*} l}(\tau) d \tau\right)^{\frac{1}{q^{*}}}
$$


Observe that $q^{*}=\frac{2 y+1}{y}$ implies $q=\frac{2 y+1}{y+1}$ and then $1-q \alpha=1-\frac{2 y+1}{y+1} \frac{1}{y+1}=\frac{y^{2}}{(y+1)^{2}}>0$. Consequently, Lemma 2.5 applies giving

$$
I_{1} \leq K_{1} t^{-\alpha}\left(\int_{0}^{t} g_{1}^{q^{*}}(\tau) E_{w}^{q^{*} l}(\tau) d \tau\right)^{\frac{1}{q^{*}}}
$$

and the theorem is proved

Remark 4.1. Observe that the conditions $k_{i}>m_{2}-\frac{1}{q^{*}} \quad(i=1,2,3,4)$ may be relaxed. Indeed, according to the proof, we only need $k_{1}>\frac{1}{q^{*}}, k_{2}>\eta-\frac{1}{q^{*}}, k_{3}>\sigma-\frac{1}{q^{*}}$ and $k_{4}>\rho-\frac{1}{q^{*}}$.

\section{References}

[1] Butler, G and T. Rogers: A generalization of a lemma of Bihari and applications to pointwise estimates for integral equations. J. Math. Anal. Appl. 33 (1971), 77 - 81.

[2] Fitzgibbon, W. E., Langlais, M., Parrott, M. E. and G. F. Webb: A diffusive system with age dependence modeling FIV. Nonlin. Anal.: Theory, Meth. \& Appl. 25 (1995), $975-$ 988.

[3] Haraux, A. and M. Kirane: Estimations $C^{1}$ pour des problèmes paraboliques semilinéaires. Ann. Fac. Sci. Toulouse 5 (1983), $265-280$.

[4] Haraux, A. and A. Youkana: On a result of K. Masuda concerning reaction-diffusion equations. Tôhoku Math. J. 40 (1988), 159 - 163.

[5] Henry, D.: Geometric Theory of Semilinear Parabolic Equations. Lect. Notes Math. 840 (1981).

[6] Hoshino, H.: On the convergence properties of global solutions for some reaction diffusion systems under Neumann boundary conditions. Diff. Int. Equ. 9 (1996), $761-778$.

[7] Hoshino, H. and Y. Yamada: Solvability and smoothing effect for semilinear parabolic equations. Funkcialaj Ekvacioj 34 (1994), 475 - 494.

[8] Hoshino, H. and Y. Yamada: Asymptotic behavior of global solutions for some reactiondiffusion systems. Nonlin. Anal.: Theory, Meth. \& Appl. 23 (1994), 639 - 650.

[9] Kirane, M. and N-E Tatar: Global existence and stability of some semilinear problems. Arch. Math. 36 (2000), $33-44$.

[10] Medved', M.: A new approach to an analysis of Henry type integral inequalities and their Bihari type versions. J. Math. Anal. Appl. 214 (1997), 349 - 366.

[11] Medved', M.: Singular integral inequalities and stability of semilinear parabolic equations. Arch. Math. (Brno) 24 (1998), 183 - 190.

[12] Michalski, M. W.: Derivatives of Noninteger Order and Their Applications (Diss. Math.). Warszawa: Polska Akad. Nauk, Inst. Mat. 1993.

[13] Rothe, F.: Global Solutions of Reaction-Diffusion Systems. Lect. Notes Math. 1072 (1983).

[14] Webb, G. F.: A reaction-diffusion model for a deterministic diffusive epidemic. J. Math. Anal. Appl. 84 (1981), 150 - 161. 